\title{
The Biology Roadmap and Its Progress of Biomedical Research in China
}

\author{
Xie GenTan ${ }^{1}$, Lin $\mathrm{Na}^{2}$, Bi ZhiLi ${ }^{3}$, Shan $\mathrm{Jie}^{4}$, Li HongMei ${ }^{5}$ \\ Binzhou Polytechnic, Binzhou, Shandong 256600, China \\ Email:11258618518@qq.com, ${ }^{2}$ bz,3383102@163.com, ${ }^{3}$ beelili@126.com
}

\begin{abstract}
The National Institutes of Health $(\mathrm{NH})$ is one of the most important medical research centers in the world. It is an executive agency of the U.S. Department of health and public health. The focus of health actions and measures in the United States and even the world are gathered here. N1h includes 27 research institutes and centers with 18000 employees and an annual budget of more than $\$ 27$ billion. In the past completed human genome sequence map and many new discoveries in biomedicine, $\mathrm{NH}$ - straight hair plays an important role. In the 21st century, life science is developing at an unprecedented pace. With its unique reputation and status, $\mathrm{NH}$ put forward a grand plan for biomedical development in the new century in 2003 NH roadmap.

Keywords: National Institutes of health, biology, Medical Science, Research plan
\end{abstract}

\section{INTRODUCTION}

With the completion of the human genome project, the development of large-scale bioassay technologies such as genomics and proteomics, and the wide application of other high technologies in the biological field, biomedical research is facing unprecedented opportunities. At the same time, the high complexity of biological system is still a great challenge for us. At present, it is urgent to link up the "gap" fields of biomedical research, and the work in these fields cannot be completed by one Institute of $\mathrm{n} 1 \mathrm{~h}$ alone. Only a combination of multiple institutes and disciplines can exert the greatest impact on medical research[1]. So what are the most pressing challenges? What are the obstacles to progress? What must we do to overcome these obstacles? What cannot be completed by a single institute, but by the strength of $\mathrm{NH}$ as a whole? In May 2002, the new director of $\mathrm{NH}$, Dr. zeihun, held a meeting immediately after a week in office to explore the answers to these problems and solutions.

After a series of discussions by more than 300 authoritative people from academic, industrial, government and social circles from 2002 to 2003 , the $\mathrm{NH}$ roadmap was gradually formed, which was composed of 28 subprogrammes of three themes (exploring new approaches, future research team and rebuilding clinical research system). In order to support the implementation of the roadmap, $\mathrm{NH}$ allocated 129 million US dollars in 2004 and 2.1 billion US dollars in 2009, all of which come from the respective research funds of $\mathrm{NH}$ Research Institute and center. For the first time in history, every institute and center is willing to raise money for the NH roadmap.

\subsection{Related Work}

$\mathrm{NH}$ roadmap is a blueprint of biomedical research in the 21 st century[2]. It will accelerate basic discovery by innovative means, and the ultimate goal is to promote the transformation of medical science knowledge and technology into people's practical interests. On September 30, 2003, President of NH [zemounf] published the roadmap. The project will deepen people's understanding of biology, encourage interdisciplinary teamwork, rebuild clinical research system, accelerate new medical discovery and improve people's health, and completely transform all biomedical research methods in NH.

This theme emphasizes the need for a deeper understanding of highly complex biological systems. This will require people to have a quantitative understanding of the interconnected networks of biomolecules and their interactions and regulation, and to accurately understand the combined changes of various molecular processes leading to diseases[3]. The implementation plan under this theme mainly includes five fields: basic biological components, biological pathways and networks, molecular library and molecular imaging, structural biology, bioinformatics and computational biology, and nanomedicine It is necessary to fully understand the basic components of biology and the process and principle from molecular interaction to biological pathway and network formation. Therefore, through a national biological network pathway technology center, NH develops the methods, technologies, instruments and reagents needed for the kinetic study of molecular interaction, so as to quantitatively measure molecular interaction at 
subcellular and short-term levels. At the same time, the technology, important reagents and data standards for proteome and metabolomics determination and analysis will be studied.

N1h will establish a small molecule library containing a large amount of compound information through its funded research center to provide organic small molecule material information for scientists studying biomolecular drugs. NH has set out to build a database of all kinds of small molecule compounds containing all the information of small molecule substances in the field of life and the interaction sites of small molecule substances[4]. The initial plan is to collect 500000 such small molecules. NH National Center for biotechnology has also established a database called PubChem, which provides the molecular structure and biological activity of compounds[5]. At the same time, NH will also support the research of molecular probe preparation technology with high specificity and high sensitivity, and increase the detection accuracy of molecular probe by 10 to 100 times. At present, $\mathrm{NH}$ has built a database of molecular probe imaging for cancer and brain, and will be expanded into a comprehensive database of probe imaging for various tissues, functions and diseases.

Mastering the spatial structure of macromolecules (especially proteins) is one of the keys to understand the nature of life activities. The first step of NH roadmap structural biology project is to build a map library of protein molecular structure in human body. This project especially emphasizes the long-standing unsolved problem of membrane protein structure determination, hoping that scientists can find a fast, effective and reliable new method to produce a large number of membrane proteins and determine their three-dimensional structure. A protein production facility for structural biology research has been developed[6]. In particular, NH proposes to develop bioinformatics and computational biology, lay an "information superhighway" for medical research and promote data sharing. $\mathrm{NH}$ also plans to set up a number of national biomedical computing centers to form a national software engineering system, so that researchers or doctors anywhere in the United States can use a set of common software for data sharing and analysis through a computer grid.In order to promote the application of nanoscience in medicine, $\mathrm{NH}$ will also set up a nanomedicine center to support the quantitative determination of biological processes at the nano level, and to design nanomaterials and nanomaterials

New medical intervention tools at molecular level, such as micro implantable drug pump, infection source or metabolic disorder sensor, etc.

The implementation plan in this field includes high-risk research, interdisciplinary research and application of public-private partnership model (6). The depth and complexity of modern biomedical research increasingly require scientists to come out of their own disciplines and encourage interdisciplinary. The $\mathrm{NH}$ roadmap will train scientists in interdisciplinary research strategies and provide cooperative development funds to enable them to engage in interdisciplinary research. The roadmap plan agrees that there can be more than one newly approved fund project lead researcher, who can be all the core members of the research team; The comprehensive evaluation of multidisciplinary research projects should be carried out

$\mathrm{NH}$ has also set up the $\mathrm{NH}$ directors pioneer awards to award biomedical researchers who dare to take risks in creative research. These methods may have a greater risk of failure, but at the same time may bring breakthrough discoveries. In addition, NH encourages the cooperation of researchers from academia, government and private sector, so as to improve public health as soon as possible[7].

The scientific research of biomedicine should be devoted to the rapid transformation of its achievements from laboratory to clinical application. The central mission of $\mathrm{NH}$ is to rapidly transform basic research into medicine, treatment and prevention methods. This mission is undoubtedly extremely challenging but crucial. $\mathrm{NH}$ hopes that patient groups, researchers, research centers, a group of qualified doctors and patients interested in testing new drugs can form a complete network; We should establish a new paradigm of clinical research information record, a new standard of clinical research protocol, a modern information technology platform for research, and a new mode and strategy of cooperation between $\mathrm{NH}$ and patient volunteers, so as to motivate clinical researchers and promote the public to participate in the research process more comprehensively.

$\mathrm{NH}$ is also preparing to establish a national electronic clinical trials and research new o * neciar to carry out clinical research Medical Informatics, promote standards and data sharing, avoid repeated experiments, and help clinical researchers better serve patients. $\mathrm{NH}$ also plans to work with other institutions to develop research report reporting standards to minimize the unnecessary formal burden that hinders research, enhance patient protection, and create a real "research community" $\left(\mathrm{H}^{\wedge}>\right.$ / / hroadmap n.gov) between patients and physicians.

\subsection{Our Contribution}

As for the $\mathrm{NH}$ road map plan, which is a brainstorming and future oriented plan, most people agree with zeihun's optimistic assessment of the road map plan. David Moore of the association of American Medical Colleges (AAMC) praised it as "a short time from planning to implementation.". Duygen thinks that it emphasizes the transformation from basic to clinical, which is beneficial to the research of new diagnosis and 
treatment methods. Compared with the human genome project, $\mathrm{f}^{\wedge} \mathrm{NCIS}$, director of the National Institute of human genome, thinks that "this is a historic moment of medical research", which has great potential. National Cancer Institute $\wedge \mathrm{NC}$. Director Andrew von Eschenbach $\wedge$ said the roadmap would help the NC hospital achieve its goal of reducing pain and death from cancer[8]. The American Academy of Environmental Health Sciences (n1ehs) is "excited and pleased" with the opportunity provided by the kenneih old network project, and believes that the advantages of n1eh are included in the roadmap. Anihon mean, director of the Department of pharmacology and cancer biology at Duke University, said the roadmap is supported because it focuses on translating basic scientific findings into patients. He said that for the first time in 30 years of scientific research, he heard the president of $\mathrm{NH}$ clearly say: "if you want to get funding from $\mathrm{NH}$, you must consider the application value of research". However, there are different views on this point of view. Some people say that they don't want to be instructed how to do scientific research. "

The NH roadmap has also met with a number of different comments[9]. Although some people are in favor of the road map plan, they are worried about its implementation. $\mathrm{D} \wedge \mathrm{ID} \mathrm{K}$ of AAMC. "Universities, scientists and magazines will all adjust to a multidisciplinary research model. The plan is in the right direction, but the change is disturbing." WiHianZ。] In the next five years, $\mathrm{NH}$ will encounter continuous budget constraints, and time will be the decisive factor for the success of $\mathrm{NH}$ roadmap. There are also doubts about whether zeihun's plans will continue beyond his term. AAMC ${ }^{\circledR}$ Tony mazzasch, vice chairman of the Research Institute of science and technology, voluntarily allocated funds from the 27 research institutes and centers of $\mathrm{NH}[10]$. He doubted whether the research institutes could support the road map when funding was tight. Some people also pointed out the problems of the road map. Some experts said that the road map did not clearly point out what to do, especially in disease prevention. David eaiony, associate dean of the school of public health and community medicine at the University of Washington, said it was unclear which traditional disease prevention studies would be suitable for the program. But davlcl Eaton $\wedge$ experts do note that support for disease prevention research is already implied in many parts of the roadmap[11]. For example, dearr $\wedge$ g pointed out that the creation of biomarkers and disease indicators can be applied to prevention research. [10]"Nothing in the roadmap is for a single study," said Kenney olden $\mathrm{cm}$. The focus of the plan is not on a specific disease, but on the mechanism of the disease.

\section{BACKGROUND}

N1h has provided funding for 192 projects in 2004, of which the first 15 projects for interdisciplinary projects have also been allocated in 2004. In addition, the request PR applications (RFA) for training scientists in emerging research fields (®) Management guidelines before and after funding have also been released ${ }^{\circ} \circ$ All the eight implementation groups except the public private cooperation implementation group have been supported by the fund in 2004 and started to implement the plan[12]. The nanomedicine center will be established this year, and other programs will start after 2005.

The institutes and centers have responded positively to this six-year blueprint. For example, researchers in the Department of pharmacology and cancer biology of DUK university received seven roadmap funds in 2004, while researchers outside $\mathrm{NH}$ were more enthusiastic about each roadmap than ever before. 1000 researchers applied for nine pioneer funds, each of which provided us $\$ 500000$ a year for five years[13]. At the same time, the NH road map program has been supported by Congress.

$\mathrm{NH}$ National Institute of allergy and infectious diseases (nadyf) funded 14 contract funds in 2004 to carry out a large-scale antibody and antigen epitope development program. In the future, na1d will provide bimune epitope database and analysis resource $1 \mathrm{edb}$ for free use by scientists all over the world. From October 12 to $14 \mathrm{~s}, 2004$, nad representatives discussed the development of the project and the establishment of $1 \mathrm{edb}$ with researchers from different institutes in San Diego, California.[14].These plans will establish detailed immune response maps of several important complex antigens to assist in the development of new diagnostic methods, new immunotherapies and vaccines.

\section{CONCLUSION}

In terms of multidisciplinary research centers, such as the Eli \& $\mathrm{ED}^{\wedge}$ e lbmad Institute in Cambridge, Massachusetts, is a joint venture with the Massachusetts Institute of technology. The mission of the Institute of Technolo $\wedge$ MIT, Harvard University and the Whitehea Institute is to develop and prepare tools (such as information, reagents and analytical methods) for genomic medicine and to use them in molecular research of diseases [13].The Institute will use unified calculation and analysis mechanism to carry out research in combination with multi-disciplinary experts in chemistry, molecular biology, computer science, computational biology and medical engineering. In 
2005, the center for neurodegenerative diseases, Institute of aging, Department of pathology and laboratory medicine, University of Pennsylvania, launched a study on the link between the development and production of new drugs for neurodegenerative diseases. Some of the important drugs involved have been approved by the research center and entered clinical trials.

\section{REFERENCES}

[1] Xu, P.; Huang, H.; Xu, W., et. al., Analysis and study on inhibition effects of medical chitin fiber products to certain kinds of bacteria, Basic \& Clinical Pharmacology \& Toxicology, 125(2019)S1: 36.

[2] Geng C., Zhao Z., Xue Z.; Xu P., Xia, Y., Preparation of Ion-Exchanged TEMPO-Oxidized Celluloses as Flame Retardant Products, Molecules ,24)2019(947.

[3] Y. Zhao, R. Wang, K. Fang, Y. Tan, S. Chen, Y. Guan, L. Hao, Investigating the synergetic dispersing effect of hydrolyzed biomacromolecule Cellulase and SDS on CuPc pigment, Colloids and Surfaces B: Biointerfaces,184(2019)110568.

[4] Zeihounie medic the NH roadmap J. science 200A 302:63-72.

[5] P. Xu, N. Na, A. M. Mohamad, Investigation the application of pristine graphdiyne (GDY) and boron-doped graphdiyne (BGDY) as an electronic sensor for detection of anticancer drug, Computational and Theoretical Chemistry, 1190(2020): 112996.

[6] P. Xu, N. Na, Study on Antibacterial Properties of Cellulose Acetate Seawater Desalination Reverse-Osmosis Membrane with Graphene Oxide, Journal of Coastal Research, 105(2020)246-251.

[7] H. Li, P. Xu, D. Liu, J. He, H. Zu, J. Song, J. Zhang, F. Tian, M. Yun and F. Wang, Low-voltage and fast-response $\mathrm{SnO} 2$ nanotubes/perovskite heterostructure photodetector, Nanotechnology, 32(2021)375202.

[8] Wangle $4 \mathrm{Roa}^{\wedge}$ nap gives new direction to trans_ NH research j. J Natl Cancer Inst 200a 95. 1741.

[9]Check; E NH' roadmapz charts course to tackle big research issues J. Nattnp 2003 425: 438

[10] P. Xu; N. Na; S. Gao; C. Geng, Determination of sodium alginate in algae by near-infrared spectroscopy, Desalination and Water Treatment, 168(2019)117-122.

[11] P. Xu, N. Na, A. M. Mohamad, Investigation the application of pristine graphdiyne (GDY) and boron-doped graphdiyne (BGDY) as an electronic sensor for detection of anticancer drug, Computational and Theoretical Chemistry, 1190(2020): 112996.
[12] P. Xu, N. Na, Study on Antibacterial Properties of Cellulose Acetate Seawater Desalination Reverse-Osmosis Membrane with Graphene Oxide, Journal of Coastal Research, 105(2020)246-251.

[13] M. Luan,F. Song, S. Qu, X. Meng, et.al.,Multi-omics integrative analysis and survival risk model construction of non-small cell lung cancer based on The Cancer Genome Atlas datasets, Oncology letters,20(2020)58. 